\title{
NEW FAMILIES OF GRAPHS DETERMINED BY THEIR GENERALIZED SPECTRUM
}

\author{
FENJIN LIU, JOHANNES SIEMONS, WEI WANG
}

\begin{abstract}
We construct infinite families of graphs that are determined by their generalized spectrum. This construction is based on new formulae for the determinant of the walk matrix of a graph. All graphs constructed here satisfy a certain extremal divisibility condition for the determinant of their walk matrix. ${ }^{1}$
\end{abstract}

\section{INTRODUCTION}

Let $G$ be a graph on the vertex set $V$ with adjacency matrix $A$ and let $\bar{G}$ be its complement, with adjacency matrix $\bar{A}$. Then the spectrum $\operatorname{spec}(G)$ of $G$ is the set of all eigenvalues of $A$, with corresponding multiplicities. It has been a longstanding problem to characterize graphs that are determined by their spectrum, that is to say: If $\operatorname{spec}(H)=\operatorname{spec}(G)$, does it follow that $H$ is isomorphic to $G$ ? If this is the case then $G$ is determined by its spectrum, or a DS graph for short. In $[4,8,9]$ it is conjectured that almost all graphs are DS, more recent surveys can be found in [5].

A variant of this problem concerns the generalized spectrum of $G$ which is given by the pair $(\operatorname{spec}(G), \operatorname{spec}(\bar{G}))$. In this situation $G$ is said to be determined by its generalized spectrum, or a DGS graph for short, if $(\operatorname{spec}(H), \operatorname{spec}(\bar{H}))=(\operatorname{spec}(G), \operatorname{spec}(\bar{G}))$ implies that $H$ is isomorphic to $G$. For the most recent results on DGS graphs see [13] and [20] in particular. To date only a few families of graphs are known to be DS or DGS. This includes some almost complete graphs [1], pineapple graphs [10] and kite graphs [11]. These are all known to be DS, and in particular DGS. In addition, all rose graphs are determined by their Laplacian spectrum except for two specific examples, see [12].

In this paper we construct infinite sequences of DGS graphs from certain small starter graphs. This construction involves the walk matrix of a graph and recent results in [20]. Let $n$ be the number of vertices of $G$ and let $e$ be the column vector of height $n$ with all entries equal to 1 . Then the walk matrix of $G$ is the $n \times n$ matrix

$$
W=\left[e, A e, A^{2} e, \cdots, A^{n-1} e\right]
$$

Date: Version 3 September 2018, printed January 14, 2019.

${ }^{1}$ Keywords: Graph spectrum, Walk matrix, Graphs determined by generalized spectrum Mathematics Subject Classification: 05C50, 15A18 
formed by the column vectors $A^{i} e$. In this paper we investigate the determinant of the walk matrix of $\bar{G}$ and of graphs obtained by joins and unions of $G$ with a single new vertex.

We prove that the determinant of the walk matrix of $G$ and that of $\bar{G}$ are the same up to a sign, see Theorem 2.1. We consider a graph $G$ and a new vertex $w$. In this situation the union $G \cup w$ and the join $G \vee w$ graphs can be defined, see Section 2. In Theorem 2.3 we obtain a formula for the determinant of their walk matrices in terms of the determinants of $G, \bar{G}$ and $W$.

In Wang [19] it is shown that $2^{\left\lfloor\frac{n}{2}\right\rfloor}$ divides $\operatorname{det}(W)$. Furthermore, if

$$
\mathcal{C}: \quad \frac{\operatorname{det}(W)}{2^{\left\lfloor\frac{n}{2}\right\rfloor}} \text { is odd and square-free }
$$

then $G$ is determined by its generalized spectrum [20]. This shows that the divisor $2^{\left\lfloor\frac{n}{2}\right\rfloor}$ plays a special role for the determinant of the walk matrix, in some sense $\mathcal{C}$ is an extremal divisibility condition, see also Section 6.2 in [13].

In Section 3 we give the construction of infinite families of graphs in which $\mathcal{C}$ holds, this is Theorems 3.3 and 3.4. This construction provides in particular infinite families of graphs which are determined by their generalized spectrum. Unlike constructing graphs with the same generalized spectrum (e.g. GM-Switching), very few methods are known for building DGS graphs. The results in this paper therefore contribute to our understanding of DGS graphs, they also give a partial answer a problem posed in [13].

All graphs in this paper are finite, simple and undirected. Our notation follows the standard texts, for instance $[3,6]$. The vertex set of the graph $G$ is denoted $V$. The characteristic polynomial $P(x)$ of $G$ is the characteristic polynomial of $A$, thus $P(x)=\operatorname{det}(x I-A)$, and the eigenvalues of $G$ or $A$ are the roots of $P(x)$. When it is necessary to refer to a particular graph $H$ we denote its vertices, adjacency or walk matrix by $V(H), A(H)$ or $W(H)$, etc.

\section{The DETERminant of THE WALK MATRiX}

We begin by giving a formula for the determinant of the walk matrix of a graph and its complement.

Theorem 2.1. Let $G$ be a graph on $n$ vertices with walk matrix $W$. Then the walk matrix of its complement satisfies

$$
\operatorname{det}(\bar{W})=(-1)^{\frac{n(n-1)}{2}} \operatorname{det}(W)
$$


Proof. Let $A$ and $\bar{A}$ be the adjacency matrix of $G$ and $\bar{G}$ respectively. We show that for each $k$ the $k$-th column of $\bar{W}$ can be expressed as a linear combination of the first $k$ columns of $W$. This is true for the first and second columns of $\bar{W}$ since

$$
\bar{A} e=(J-I-A) e=(n-1) e-A e,
$$

where $J$ is the all-one matrix and $I$ is the identity matrix. So we assume that the claim holds for some $k$, that is, there exist numbers $c_{0}, c_{1}, \ldots, c_{k-1} \in \mathbb{R}$ such that

$$
\bar{A}^{k-1} e=\sum_{i=0}^{k-1} c_{i} A^{i} e .
$$

Since $J A^{i} e=\left(e^{T} A^{i} e\right) e$, we have

$$
\begin{aligned}
\bar{A}^{k} e & =\bar{A}\left(\bar{A}^{k-1} e\right) \\
& =(J-I-A)\left(\sum_{i=0}^{k-1} c_{i} A^{i} e\right) \\
& =\left[\sum_{i=0}^{k-1} c_{i}\left(e^{T} A^{i} e\right)-c_{0}\right] e-\sum_{i=1}^{k-1}\left(c_{i-1}+c_{i}\right) A^{i} e-c_{k-1} A^{k} e .
\end{aligned}
$$

Therefore the above claim is true. In particular, for each $k=1,2, \ldots$, we conclude that the coefficient of the vector $A^{k} e$ is $-c_{k-1}=(-1)^{k}$. Substituting (1) into $\bar{W}$ gives

$$
\operatorname{det}(\bar{W})=\operatorname{det}\left[e,-A e, A^{2} e,-A^{3} e, \cdots,(-1)^{n-1} A^{n-1} e\right]
$$

and so the result follows from the multilinearity of the determinant.

By the same reasoning we have the following result for the leading principal submatrices of the walk matrix.

Corollary 2.2. Let $W_{1}, W_{2}, \ldots, W_{k}$ (resp. $\bar{W}_{1}, \bar{W}_{2}, \ldots, \bar{W}_{k}$ ) be the first $k$ leading principal submatrices of the walk matrix $W$ (resp. $\bar{W}$ ) for $k=1,2, \ldots, n$. Then

$$
\operatorname{det}\left(\bar{W}_{k}\right)=(-1)^{\frac{k(k-1)}{2}} \operatorname{det}\left(W_{k}\right) \text {. }
$$

The graph $G$ is controllable if its walk matrix $W(G)$ is invertible. This property can also be characterized by the main eigenvalues and main eigenvectors of the graph, see [7]. The relevance of controllability becomes clear from the recent work of O'Rourke and B. Touri [14] who proved Godsil's conjecture [7] that asymptotically all graphs are controllable. We note that according to Theorem 2.1 a graph $G$ is controllable if and only if its complement $\bar{G}$ is controllable.

Let $G$ be a graph and let $w$ be a new vertex, $w \notin V(G)$. Then the union of $G$ and the singleton graph $\{w\}$, denoted by $G \cup w$, is the graph obtained from $G$ by adding $w$ as 
an isolated vertex. The join of $G$ and $\{w\}$, denoted by $G \vee w$, is the graph obtained from $G$ by adding the vertex $w$ and making it adjacent to all vertices of $G$. For these graph operations we have the following result.

Theorem 2.3. Let $G$ be a graph with adjacency matrix $A$ and walk matrix $W$. Then we have

(i) $\operatorname{det}(W(G \cup w))= \pm \operatorname{det}(A) \operatorname{det}(W)$ and

(ii) $\operatorname{det}(W(G \vee w))= \pm \operatorname{det}(\bar{A}) \operatorname{det}(W)$.

The sign only depends on the position of the new vertex. For instance, if $\left\{w, v_{1}, \ldots, v_{n}\right\}$ are the vertices of $G \cup w$ then $\operatorname{det}(W(G \cup w))=\operatorname{det}(A) \operatorname{det}(W)$ and $\operatorname{det}(W(G \vee w))=$ $\operatorname{det}(\bar{A}) \operatorname{det}(W)$.

Proof. Denote by $W^{\prime}=A\left[e, A^{2} e, \cdots, A^{n-1} e, A^{n} e\right]$. Then, taking the new vertex $w$ as the first vertex in $G \cup\{w\}$, we have

$$
W(G \cup w)=\left[\begin{array}{cc}
1 & 0_{n \times 1} \\
1_{n \times 1} & W^{\prime}
\end{array}\right] .
$$

Expanding the determinant of $W(G \cup w)$ along the first row gives

$$
\operatorname{det}(W(G \cup w))=\operatorname{det}\left(W^{\prime}\right)=\operatorname{det}(A W)=\operatorname{det}(A) \operatorname{det}(W) .
$$

If the new vertex $w$ is labelled even, then $\operatorname{det}(W(G \cup w))=-\operatorname{det}(A) \operatorname{det}(W)$. This proves the first part of the theorem.

For the second part, first note that $G \vee w=\overline{\bar{G} \cup w}$. Now use Theorem 2.1 to obtain

$$
\begin{aligned}
\operatorname{det}(W(G \vee w)) & =\operatorname{det}(W(\overline{\bar{G} \cup w)}) \\
& =(-1)^{\frac{n(n+1)}{2}} \operatorname{det}(W(\bar{G} \cup w)) \\
& = \pm \operatorname{det}(\bar{A}) \operatorname{det}(\bar{W}) \\
& = \pm \operatorname{det}(\bar{A}) \operatorname{det}(W) .
\end{aligned}
$$

This completes the proof.

Remark 1. A graph is singular if its adjacency matrix is singular, see [16, 17]. The theorem has an interesting consequence for singular graphs: If $G$ is controllable then $G \cup w$ is controllable if and only if $G$ is not singular. Similarly, if $G$ is controllable then $G \vee w$ is controllable if and only if $\bar{G}$ is not singular. 


\section{Constructing DGS graphs}

In this section we construct families of DGS graphs by using the union and join operations. We begin with the Coefficient Theorem of Sachs. It relates the coefficients of the characteristic polynomial of the graph to its structure. An elementary graph is a graph in which each component is $K_{2}$ or a cycle.

Theorem 3.1 (Sachs Coefficients Theorem, see e.g. [3]). Let $G$ be a graph on $n$ vertices with characteristic polynomial $P(x)=x^{n}+c_{1} x^{n-1}+\cdots+c_{n-1} x+c_{n}$. Denote by $\mathcal{H}_{i}$ the set of all elementary subgraphs of $G$ with $i$ vertices. For $H$ in $\mathcal{H}_{i}$ let $p(H)$ denote the number of components of $H$ and $c(H)$ the number of cycles in $H$. Then

$$
c_{i}=\sum_{H \in \mathcal{H}_{i}}(-1)^{p(H)} 2^{c(H)}, \quad \text { for all } i=1, \ldots, n .
$$

The following theorem due to Wang [20] characterizes certain DGS graphs by an arithmetic property of the determinant of their walk matrix.

Theorem 3.2 (Wang [20]). Let $G$ be a graph on $n$ vertices with $n \geq 6$ and walk matrix $W$. Then $2^{\left\lfloor\frac{n}{2}\right\rfloor}$ divides $\operatorname{det}(W)$. Furthermore, if $2^{-\left\lfloor\frac{n}{2}\right\rfloor} \operatorname{det}(W)$ is odd and square-free then $G$ is determined by is generalized spectrum.

We are now able to state our next result.

Theorem 3.3. Let $G_{0}, G_{1}, G_{2}, \ldots$ be a sequence of graphs which satisfy the following conditions

$$
G_{i}= \begin{cases}G_{i-1} \cup w_{i} & (\text { if } i \geq 1 \text { is odd }) \\ G_{i-1} \vee w_{i} & (\text { if } i \geq 1 \text { is even })\end{cases}
$$

Denote $n_{0}:=\left|V\left(G_{0}\right)\right|, \quad a:=\left|\operatorname{det}\left(A\left(G_{0}\right)\right)\right|, \quad b:=\left|\operatorname{det}\left(W\left(G_{0}\right)\right)\right|$ and $p:=\left|\operatorname{det}\left(A\left(\bar{G}_{1}\right)\right)\right|$. Then

$$
\left|\operatorname{det}\left(W\left(G_{i}\right)\right)\right|=a^{\left\lceil\frac{i}{2}\right\rceil} b p^{\left\lfloor\frac{i}{2}\right\rfloor} \text { for all } i \geq 1 \text {. }
$$

Proof. By Theorem 2.3 (i) and (ii) we have

$$
\begin{aligned}
& \left|\operatorname{det}\left(W\left(G_{1}\right)\right)\right|=\left|\operatorname{det}\left(W\left(G_{0} \cup w_{1}\right)\right)\right|=\left|\operatorname{det}\left(A\left(G_{0}\right)\right) \operatorname{det}\left(W\left(G_{0}\right)\right)\right|=a b, \\
& \left|\operatorname{det}\left(W\left(G_{2}\right)\right)\right|=\left|\operatorname{det}\left(W\left(G_{1} \vee w_{2}\right)\right)\right|=\left|\operatorname{det}\left(A\left(\bar{G}_{1}\right)\right) \operatorname{det}\left(W\left(G_{1}\right)\right)\right|=a b p .
\end{aligned}
$$

Thus the result holds for $i=1,2$. Since the determinant of the adjacency matrix and the constant term of its characteristic polynomial are the same up to sign we use Theorem 3.1 to link it to the elementary spanning subgraphs. By induction we have $\left|\operatorname{det}\left(A\left(G_{2 i}\right)\right)\right|=a$ 
and $\left|\operatorname{det}\left(A\left(\bar{G}_{2 i+1}\right)\right)\right|=p$ for any integer $i \geq 1$. Since all elementary spanning subgraphs $H\left(G_{2 i}\right)$ in $\mathcal{H}_{n_{0}+2 i}\left(G_{2 i}\right)$ must have $K_{2}=w_{2 i} w_{2 i-1}$ as a component, there is a bijection between the elementary spanning subgraph set $\mathcal{H}_{n_{0}+2 i}\left(G_{2 i}\right)$ and $\mathcal{H}_{n_{0}+2(i+1)}\left(G_{2(i+1)}\right)$ i.e., there is a bijection

$$
\begin{aligned}
& f: \mathcal{H}_{n_{0}+2 i}\left(G_{2 i}\right) \leftrightarrow \mathcal{H}_{n_{0}+2(i+1)}\left(G_{2(i+1)}\right) \text { with } \\
& f\left(H\left(G_{2 i}\right)\right)=H\left(G_{2 i}\right) \cup w_{2 i+2} w_{2 i+1} .
\end{aligned}
$$

Note that the component $K_{2}=w_{2 i+2} w_{2 i+1}$ only changes the sign of the constant coefficient of the characteristic polynomial. Therefore each pair of elementary spanning subgraphs $H\left(G_{2 i}\right)$ and $H\left(G_{2 i}\right) \cup w_{2 i+2} w_{2 i+1}$ contribute opposite signs to the constant terms of $P_{G_{2 i}}(x)$ and $P_{G_{2(i+1)}}(x)$, respectively. Hence $\left|\operatorname{det}\left(A\left(G_{2(i+1)}\right)\right)\right|=a$. Analogously we have $\left|\operatorname{det}\left(A\left(\bar{G}_{2 i+1}\right)\right)\right|=p$. Equation (2) follows by applying Theorem 2.3 repeatedly.

From this result we obtain infinite sequences of graphs that are determined by their generalized spectrum:

Theorem 3.4. Let $G_{0}, G_{1}, G_{2}, \ldots$ be as in Theorem 3.3 and denote $\left|V\left(G_{0}\right)\right|$ by $n_{0}$. Suppose that $\{a, p\}=\{1,2\}$ and that $b \cdot 2^{-\left\lfloor\frac{n_{0}}{2}\right\rfloor}$ is an odd square-free integer. Then the graphs $G_{i}$ are determined by their generalized spectrum, for all $i \geq 1$.

Proof. It is easy to see that $2^{-\left\lfloor\frac{n_{0}+i}{2}\right\rfloor} \cdot \operatorname{det}\left(W\left(G_{i}\right)\right)$ is odd and square-free when $\{a, p\}=$ $\{1,2\}$ and $b \cdot 2^{-\left\lfloor\frac{n_{0}}{2}\right\rfloor}$ is an odd square-free integer. Thus our result follows from Theorem 3.2 and Theorem 3.3 .

We mention several remarks and open problems.

Remark 2. In the introduction we discussed the importance of the property $\mathcal{C}$ as an extremal divisibility condition for the walk matrix of a graph. All the graphs $G_{i}$ in Theorem 3.4 now satisfy the condition $\mathcal{C}$.

Remark 3. There are indeed many graphs $G_{0}$ that are suitable starters for such sequences. Among the 112 connected graphs on six vertices there are 8 controllable graphs, labelled 59,77 in [2] with $|\operatorname{det}(W)|=3 \cdot 2^{3}$ and $46,60,67,85,87,98$ in [2] with $|\operatorname{det}(W)|=2^{3}$. These graphs therefore have property $\mathcal{C}$ and are controllable. According to Theorems 3.2, 3.3 and 3.4 we obtain infinite series of graphs based on 6 of these 8 graphs as an initial $G_{0}$, namely graphs labelled $59,77,67,85,87,98$. In particular, all graphs in these series have property $\mathcal{C}$ and hence are DGS graphs. 
Remark 4. Mao et al. [13] stated the problem of characterizing graphs with $|\operatorname{det}(W)|=$ $2^{\left\lfloor\frac{n}{2}\right\rfloor}$. This condition can be viewed as a strengthening of condition $\mathcal{C}$ and is worth investigation independently. By Theorems 3.3 and 3.4 we can construct infinite families of such graphs based the controllable graphs labelled $67,85,87,98$ in [2].

Remark 5. Theorems 2.1, 3.2 and 3.4 imply that the complement of any $G_{i}$ constructed in Theorem 3.3 is also DGS, for all $i \geq 0$.

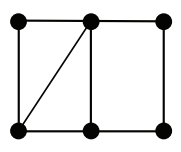

$G_{0}$

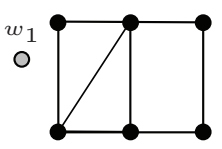

$G_{1}$

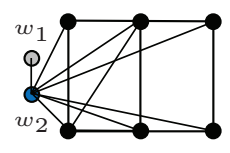

$G_{2}$

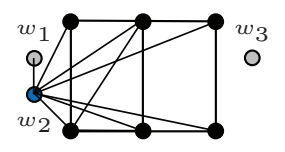

$G_{3}$

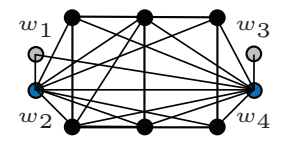

$G_{4}$

FiguRE 1. Constructing a family of DGS graphs from $G_{0} \cong \# 67$

Remark 6. Let $G_{0}$ be the graph labelled 67 in [2], see Fig.1. We show schematically the first five DGS graphs obtained from Theorem 3.3. It is easy to verify that these graphs satisfy $|\operatorname{det}(W)|=\left\lfloor\frac{n}{2}\right\rfloor$.

Open Problems. We conclude with two open problems:

(i) Find other constructions of graphs satisfying property $\mathcal{C}$.

(ii) Determine the generalized spectrum of graphs with property $\mathcal{C}$ and classify such graphs.

Acknowledgements. The first author would like to express his gratitude to Professor Qiongxiang Huang for guiding him towards Spectral Graph Theory and the faculty of the School of Mathematics at UEA for accepting him into their friendly research environment.

This work is supported by the National Natural Science Foundation of China (Nos. 11401044, 11471005, 11501050), Postdoctoral Science Foundation of China (No. 2014M560754), Postdoctoral Science Foundation of Shaanxi, the Fundamental Research Funds for the Central Universities (No. 300102128201) and the Foundation of China Scholarship Council (No. 201706565015).

\section{REFERENCES}

[1] M. Cámara, W.H. Haemers, Spectral characterizations of almost complete graphs, Discrete Appl. Math. 176 (2014) 19-23.

[2] D.M. Cvetković, M. Petrić, A table of connected graphs on six vertices, Discrete Math. 50 (1984) 37-49.

[3] D.M. Cvetković, P. Rowlinson, S.K. Simić, An Introduction to the Theory of Graph Spectra, Cambridge University Press, Cambridge, 2010. 
[4] E.R. van Dam, W.H. Haemers, Which graphs are determined by their spectrum, Linear Algebra Appl. 373 (2003) 241-272.

[5] E.R. van Dam, W.H. Haemers, Developments on spectral characterizations of graphs, Discrete Math. 309 (2009) 576-586.

[6] C.D. Godsil, G. Royle, Algebraic Graph Theory, Springer-Verlag, New York, 2001.

[7] C.D. Godsil, Controllable subsets in graphs, Ann. Comb. 16 (2012) 733-744.

[8] C.D. Godsil, Are almost all graphs determined by their spectrum? https://pdfs. semanticscholar.org/presentation/6b04/5c86e2212ac72e2e80024b025d76bf52c72d.pdf

[9] W.H. Haemers, Are almost all graphs determined by their spectrum? http://members. upc.nl/w.haemers/sams.pdf

[10] T. Hatice, S. Sorgun, W.H. Haemers, On the spectral characterization of pineapple graphs, Linear Algebra Appl. 507 (2016) 267-273.

[11] T. Hatice, S. Sorgun, The kite graph is determined by its adjacency spectrum, Appl. Math. Comput. 330 (2018) 134-142.

[12] C.X. He, E.R. van Dam, Laplacian spectral characterization of roses, Linear Algebra Appl., 536 (2018) 19-30.

[13] L.H. Mao, F.J. Liu, W. Wang, A new method for constructing graphs determined by their generalized spectrum, Linear Algebra Appl., 477 (2015) 112-127.

[14] S. O'Rourke, B. Touri, On a conjecture of Godsil concerning controllable random graphs, Siam J. Control Optim. 54 (2016) 3347-3378.

[15] P. Rowlinson, The main eigenvalues of a graph: a survey. Appl. Anal. Discrete Math. 1 (2007) 445-471.

[16] I. Sciriha, A characterization of singular graphs, Electronic Journal of Linear Algebra 16 (2007) 451-462.

[17] J.Siemons, A.Zalesski, Remarks on singular Cayley graphs and vanishing elements of simple groups, arXiv: 1804.01204, J Algebr Comb DOI 10.1007/s10801-018-0860-0, (2018).

[18] W. Wang, C.X. Xu, A sufficient condition for a family of graphs being determined by their generalized spectra, Eur. J. Combin. 27 (2006) 826-840.

[19] W. Wang, Generalized spectral characterization revisited, Electron J. Comb. 20 (4) (2013) \# P4.

[20] W. Wang, A simple arithmetic criterion for graphs being determined by their generalized spectra, J. Combin. Theory Ser. B 122 (2017) 438-451.

F. Liu: School of Science, Chang'an University, Xi'an, P.R. China, 710046

School of Mathematics and Statistics, Xi'an Jiaotong University, Xi'An, P.R. China, 710049 School of Mathematics, University of East Anglia, Norwich, Norfolk, NR4 7TJ, UK. 
J. Siemons: School of Mathematics, University of East Anglia, Norwich, NR4 7TJ, UK

E-mail address: j.siemons@uea.ac.uk

W. Wang: School of Mathematics and Statistics, Xi’an Jiaotong University, Xi'an, P.R. China, 710049

E-mail address: wang_weiw@163.com 